
PHARMACEUTICAL SCIENCES

\title{
QUALITY MEASURES IN SURGICAL PALLIATIVE CARE ADAPTING EXISTING PALLIATIVE CARE MEASURES TO IMPROVE CARE FOR SERIOUSLY ILL SURGICAL PATIENTS
}

${ }^{1}$ Dr Maleeha Mujeeb Khan, ${ }^{2}$ Dr Hafiz Sami Ullah, ${ }^{3}$ Dr Umar Iqbal

${ }^{1}$ WMO,Punjab Rangers Teaching Hospital,Lahore, ${ }^{2} \mathrm{MO}, \mathrm{DHQ}$ Hospital,Kasur, ${ }^{3} \mathrm{MO}, \mathrm{DHQ}$ Hospital,Kasur.

\begin{tabular}{|l|l||}
\hline \hline Article Received: February $2019 \quad$ Accepted: March 2019 \\
\hline \hline Abstract: \\
Surgeons are critical providers for the growing number of seriously ill patients, many who live with burdensome, \\
life-limiting chronic conditions that impose severe functional limitations or carry a high risk of mortality. Over \\
500,000 older adults, many with frailty and cognitive impairment, undergo high-risk surgery annually and nearly \\
$20 \%$ of Medicare decedents undergo an inpatient surgical procedure in the last month of life.1 Older and seriously \\
ill patients invariably suffer from higher rates of mortality and morbidity after elective and emergency surgery \\
compared to their younger counterparts.2,3 In addition, many older patients experience distressing physical and \\
psychosocial symptoms, and functional impairments, and reduced quality of life (QOL) after surgery. As such, these \\
patients are also at a high risk of dissatisfaction with care and receiving care discordant with their overall health \\
goals. \\
Keywords: End of life care, palliative care research, quality measurement, serious illness, surgical patients \\
\hline \hline
\end{tabular}

Corresponding author:

Dr. Maleeha Mujeeb Khan,

WMO,Punjab Rangers Teaching Hospital,Lahore.

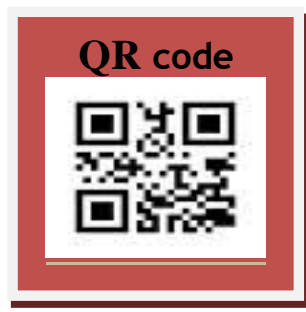

Please cite this article in press Maleeha Mujeeb Khan et al., Quality Measures In Surgical Palliative Care Adapting Existing Palliative Care Measures To Improve Care For Seriously Ill Surgical Patients., Indo Am. J. P. Sci, 2019; 06(04). 


\section{INTRODUCTION:}

Palliative care (PC) is an interdisciplinary approach to care focused on improving QOL for patients and families at all stages of serious illness and improves the quality of care for seriously ill patients. Primary PC delivered by surgical teams is intended to be delivered alongside routine surgical care and includes physical and psychosocial symptom management and establishing treatment goals and preferences. Specialist PC delivered by PC teams includes complex symptom management, conflict resolution regarding goals of treatment, or exploring options for hospice. In this Perspective, the term PC is used broadly to address PC delivery to surgical patients regardless of who is delivering PC. Seriously ill patients have a significant burden of PC needs that can be exacerbated by an acute or elective surgical episode. PC interventions in surgical patients are associated with improvements in outcomes that better reflect the patient experience, such as better QOL, decreased symptom burden, and improved patientreported quality of communication. These interventions decrease hospital and intensive care unit (ICU) length of stay without increased mortality. Despite these benefits, PC in surgery remains poorly defined. A major barrier to integrating PC into surgical care for seriously ill patients has been a lack of standardized quality measures to consistently measure PC delivery. Surgical quality improvement programs, such as the National Surgical Quality Improvement Program (NSQIP), have averted hundreds of thousands of deaths and complications in the last 2 decades by measuring surgical outcomes and benchmarking hospitals based on performance. The success of NSQIP sets a precedent for quality measurement in surgery, which can be leveraged to improve PC delivery to seriously ill surgical patients.

Recent efforts to test new geriatric-specific variables through the NSQIP Geriatric Surgery Pilot Project, such as preoperative cognitive status and functional status on discharge, highlight a national interest in evaluating care delivery relevant to seriously ill surgical patients. Nonetheless, these new measures are not inclusive enough to neither encompass the needs of all seriously ill surgical patients nor specifically address PC needs across the entire surgical episode from the preoperative phase to post discharge. Furthermore, NSQIP does not presently assess outcomes such as symptom improvement or improved QOL that are important in PC. Therefore, a significant gap remains between the current standards of quality measurement in surgery and quality measures that patients value. PC quality measures exist in other specialties such as critical care medicine, cardiology, and oncology; however, efforts to integrate PC quality measures into surgery are lacking. We hypothesize that although few quality measures exist to measure PC delivery to surgical patients, existing PC measures in other settings could be adapted or applied to surgery. These findings can provide a framework for surgeons, professional organizations, and policymakers to adapt relevant measures to improve PC delivery to surgical patients. Therefore, we performed an environmental scan of existing quality measures to identify existing quality measures relevant to PC delivery in surgery.

\section{Exploring Surgical Palliative Care Measures within the Phases of Surgical Care}

Recently, the American College of Surgeons conceptualized the surgical episode over 5 phases of surgical care, including preoperative evaluation, immediate preoperative readiness, intraoperative, postoperative, and post discharge phases. We imagined that the measures identified in this environmental scan could be adapted and employed to measure PC delivery within 4 of the 5 phases as well as a separate phase for patients who die proximate to surgery. Examples of overlapping and potentially adaptable quality measures are summarized in Table 1. 


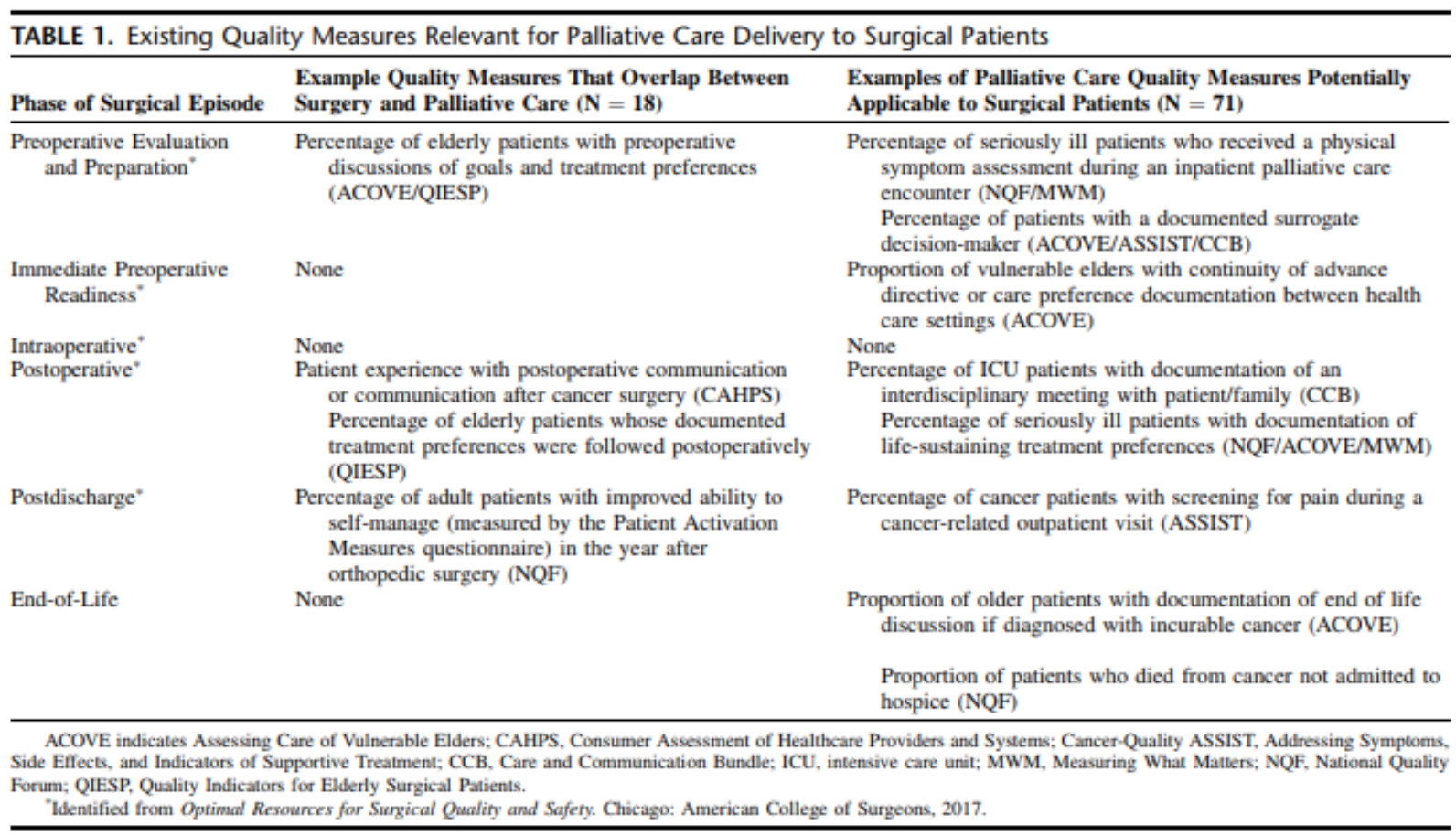

In the preoperative evaluation phase, surgeons engage with patients to deliberate surgery. Overlapping quality measures include patientreported experience with surgeon communication (CAHPS), percentage of vulnerable elders with documented preoperative discussions regarding clinical and functional risks (ACOVE), and documented discussions of advance directives (ACOVE/QIESP).

\section{DISCUSSIONS:}

Existing PC measures potentially adaptable to this phase include the percentage of patients receiving physical symptom assessments (NQF/ACOVE) or have a documented surrogate decision-maker (ACOVE). This scan revealed no overlapping quality measures for the immediate preoperative readiness phase. However, existing measures such as ensuring continuity of advance directives from outpatient to inpatient settings (ACOVE) could be adapted for this phase. In the postoperative period, overlapping measures include patient experience with postoperative communication (CAHPS), following treatment preferences after surgery among older adults (QIESP), and postoperative pain assessment and management (QIESP). Potentially adaptable quality measures include the proportion of patients with documentation of life-sustaining treatment preferences (ACOVE/NQF/MWM/CCB), family meetings in patients with prolonged postoperative
ICU stay (CCB), and physical symptom assessments (ACOVE/MWM).

After discharge, seriously ill surgical patients continue to have PC needs. Current overlapping measures are limited to self-management ability in the year after orthopaedic surgery (NQF) and mortality rates after hip fracture admissions among older adults (NQF). However, potentially adaptable PC measures include symptom assessment and management (NQF/ACOVE) in the post discharge period. Surgeons also provide EOL care to surgical patients. No surgical measures presently exist for quality EOL care. However, potentially adaptable PC quality measures include those for terminal care, such as management of dyspnea and pain (ACOVE/MWM), ICU stays near EOL for expected deaths (NQF), and bereavement services for survivors (NQF/ACOVE).

PC delivery is critical to improve value for seriously ill surgical patients. This environmental scan highlights opportunities to translate existing quality measures in related specialties to develop PC measures for surgical patients. Importantly, measures unique to surgery, such as documenting preoperative life-sustaining treatment preferences or preoperative palliative symptom assessment for palliative operations, are not captured among current measures. Even where overlapping measures exist, they do not 
necessarily address outcomes seriously ill patients' value. These findings challenge surgical leaders to use previously described approaches to adapt and create PC quality measures relevant to seriously ill surgical patients. These measures should be tested to establish baseline performance and set goals for improvement, and then ultimately integrated into quality improvement programs to improve the value of surgical care for complex seriously ill patients.

Quality measures from these sources were organized into the following categories: PC measures, surgical measures, measures that overlap between $\mathrm{PC}$ and surgery (PC measures about surgical patients or surgical measures concerning PC), and PC measures in other specialties that could potentially apply to surgical patients. Measures were defined as PC if they addressed one of the established PC domains structure and processes of care, physical and psychosocial symptom management, social, spiritual, and cultural aspects of care, care near end of life (EOL), and ethical and legal aspects of care. Measures were defined as surgical depending on the endorser's classification or if the measure specifically addressed surgery.

\section{CONCLUSION:}

Measures were identified as overlapping if the endorser classified the measure in both surgery and $\mathrm{PC}$ or if the measure addressed PC domains among surgical patients. Two authors (KCL, general surgery resident; SS, trained research coordinator) reviewed and categorized each measure and a third author (ZC, surgeon, board certified in hospice and palliative medicine) reviewed and resolved any disagreement. Among the measures reviewed, 643 surgical measures and PC measures were identified. Only quality measures overlap between $\mathrm{PC}$ and surgery. However, PC measures exist in other fields that can be adapted to measure quality $\mathrm{PC}$ in surgery. For example, the NQF-Endorsed Measure, percentage of vulnerable elders admitted to the ICU with documentation of care preferences within 48 hours, could also be applied to seriously ill patients undergoing surgery.

\section{REFERENCES:}

1. ACS NSQIP. User Guide for the 2015 ACS NSQIP Procedure-Targeted Participant Use Data File 2016.

2. Berian JR, Zhou L, Hornor MA, et al. Optimizing surgical quality datasets to care for older adults: lessons from the American College of Surgeons NSQIP Geriatric Surgery pilot. J Am Coll Surg. 2017;225:702.e1-712.e1.

3. Cooper Z, Scott JW, Rosenthal RA, et al. Emergency major abdominal surgical procedures in older adults: a systematic review of mortality and functional outcomes. J Am Geriatr Soc. 2015;63:2563-2571.

4. De RooML, Leemans K, Claessen SJ, et al. Quality indicators for palliative care: update of a systematic review. J Pain Symptom Manage. 2013;46:556-572.

5. Finks JF, Osborne NH, Birkmeyer JD. Trends in hospital volume and operative mortality for highrisk surgery. N Engl J Med. 2011;364:21282137.

6. Finlayson E, Fan Z, Birkmeyer JD. Outcomes in octogenarians undergoing highrisk cancer operation: a national study. J Am Coll Surg. 2007;205:729-734.

7. Institute of Medicine. Dying in America: Improving Quality and Honoring Individual Preferences Near the End of Life. Washington, DC: The National Academies Press; 2015.

8. Lilley EJ, Khan KT, Johnston FM, et al. Palliative care interventions for surgical patients: a systematic review. JAMA Surg. 2016;151:172183.

9. Liu JB, Pusic AL, Temple LK, et al. Patientreported outcomes in surgery: listening to patients improves quality of care. Bull Am Coll Surg. 2017; 102:19-23.

10. National Quality Forum. NQF Measures 2017. Available at: http://www.qualityforum.org/QPS/. Accessed April 6, 2019.

11. Vidri RJ, Blakely AM, Kulkarni SS, et al. American College of Surgeons National Surgical Quality Improvement Program as a qualitymeasurement tool for advanced cancer patients. Ann Palliat Med. 2015;4:200-206. 